\title{
Application-specific programming of MEMS microphones
}

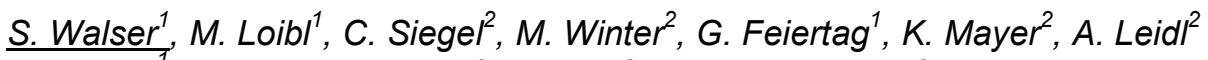 \\ ${ }^{\top}$ Munich University of Applied Sciences, Munich, Germany \\ ${ }^{2}$ EPCOS AG a TDK group company, Munich, Germany
}

\begin{abstract}
The paper presents a novel method to trim MEMS microphones towards specific applications. This method allows tuning the acoustical parameters of programmable MEMS microphones after fabrication. Depending on the customer's requirements, either a high signal to noise ratio (SNR) or a low total harmonic distortion (THD) at high sound pressure levels (SPL) can be achieved. A high SNR of up to $66 \mathrm{~dB}(\mathrm{~A})$ was reached by programming high bias voltages. Low bias voltages and a correction by gain factors allow the use at high sound pressure levels. Within a MEMS microphone fabrication batch the standard deviation of sensitivity was reduced by a factor of 5 . A comparison of the two programmed settings shows that with the high SNR setting the SNR is better by $1 \mathrm{~dB}$. The THD @ $127 \mathrm{~dB}_{\mathrm{SPL}}$ has the value of $1.0 \%$ for the high SPL setting in comparison to $2.2 \%$ for the high SNR setting.
\end{abstract}

Keywords: MEMS, microphone, program, OTP, narrow sensitivity, SNR, SPL, THD.

\section{Introduction}

In the segment of consumer electronics, microelectromechanical condenser microphones are often used as acoustic sensors [1]. A sensor chip converts an incoming sound wave into a capacitive change. An applicationspecific integrated circuit (ASIC) converts the capacitive change into an electrical audio signal. The two chips are integrated into a surface-mount device (SMD) package. Two packaging variants are used for bottom-port MEMS microphones, wire bonding packaging and flip-chip packaging [2]. During the last few years, research has focused on the design of the capacitive sensor, e.g. single-ended [3], double backplate [4] and multiple membrane systems [5]. Now, the state of the art parameters of commercial capacitive bottomport MEMS microphones are:

- $\quad$ Sensitivity: 38 dBV/Pa @ 1 kHz;

- SNR: $65 \mathrm{~dB}(\mathrm{~A})$;

- $\quad$ THD: $10 \%$ at $124 \mathrm{~dB}_{\mathrm{SPL}}$.

In addition to their good performance, the main reasons to use MEMS microphones in consumer electronic products are a small size around $3.5 \times 2.5 \times 1 \mathrm{~mm}^{3}$ and resistance to soldering heat [6].

During the last few years, audio signal processing, like noise reduction and stereo recording, have become more and more important for mobile phones. For most audio algorithms, the uniformity of the microphone sensitivities within one device is decisive [7]. For MEMS microphone fabrication this means, that it is necessary to produce microphones with a narrow sensitivity distribution. One of the high revenue segments for MEMS microphones is the mobile phone market, which currently specifies sensitivity distributions as low as $\pm 1 \mathrm{~dB}$.

The most important factors influencing the accuracy of manufactured microphones are variations of the wafer fabrication process and the packaging process. One way to compensate process tolerances is trimming the MEMS microphones after completing the fabrication process. By using a programmable ASIC, it is possible to trim MEMS microphones to a specified sensitivity [8].

One of the most important parameters for highend recording of speech and music is the SNR. A high SNR allows detecting low incoming audio signals above the noise floor. Another upcoming trend in consumer electronics is the sports and leisure segment, for example action and outdoor cameras. For these applications, a low THD at high sound pressures is necessary to record loud sounds or to avoid wind noise clipping. For microphones, there is a tradeoff between high SNR and low THD at high SPL. Increasing the dynamic range of a microphone reduces the SNR. Up to now increasing the SNR or the dynamic range requires a modification of the sensor design. For the manufacturer this means that MEMS microphones must be designed for the specific application.

This paper presents a method for tuning the SNR or the dynamic range within the same 
sensor design by using a programmable MEMS microphone. The focus lies on the measurement of SNR and the THD at high SPL for different settings of the programming parameters. The benefit of programming microphones from the same production batch is shown. It is described how the microphones can be tuned towards a high SNR with narrow sensitivity distribution and towards low THD at high SPL.

\section{Programmable MEMS microphone}

The investigations are based on a programmable condenser MEMS microphone [8]. Figure 1 shows a schematic cross section of the microphone package.

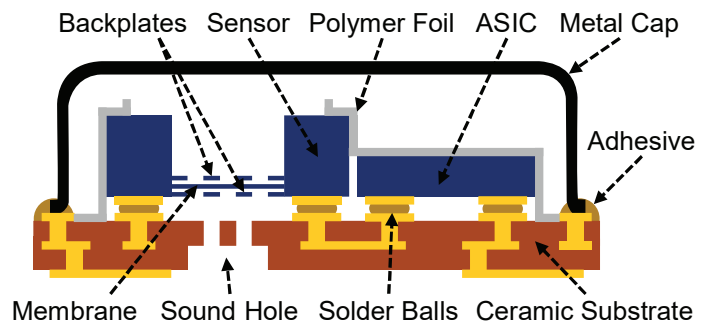

Fig. 1: Schematic cross section of a bottomport MEMS microphone in a flip-chip package with metal cap [9]: ceramic substrate with sound hole, differential sensor chip, programmable ASIC chip, separation of front-volume and back-volume by a polymer foil, large back cavity closed by a metal cap.

The microphone's SMD package $\left(3.35 \times 2.5 \times 1.0 \mathrm{~mm}^{3}\right)$ is based on flip-chip technology [2]. For the substrate a high temperature cofired ceramic (HTCC) is used. A polymer foil separates the front-volume from the back-volume. The back-volume is closed with a metal lid. Detailed information of the fabrication process is presented in [9].

Figure 2 shows the schematic of a programmable MEMS microphone. Here, a differential sensor chip with a size of $1.45 \times 1.45 \times 0.45 \mathrm{~mm}^{3}$ is used. The movable membrane, with a thickness of $0.4 \mu \mathrm{m}$, is between two perforated backplates with a thickness of $3 \mu \mathrm{m}$. The membrane and the backplates have a diameter of $1.2 \mathrm{~mm}$ and a gap distance of around $2 \mu \mathrm{m}$. The main benefit of a double backplate transducer is the differential output signal. Thereby, the SNR increases by around $3 \mathrm{~dB}(\mathrm{~A})$ [10]. Detailed investigations about double backplate microphone sensor chips are given in [11].

Within an ASIC chip $\left(1.45 \times 1.0 \times 0.2 \mathrm{~mm}^{3}\right)$, a constant preload of the membrane-backplate system is provided by a bias supply voltage. This leads to an electrical field between membrane and backplates. In the case of an incident sound pressure, the preloaded membrane moves and causes a differential capacity change. This differential capacitive change is transformed into electrical signals and transmitted via two amplifiers to the differential microphone outputs. To compensate acoustical deviations after completing the microphone fabrication process a non-volatile one-time programmable (OTP) memory is implemented on the ASIC [12]. The OTP is based on the principle of a very high cell resistance, which decreases significantly, by programming with a high voltage [12].

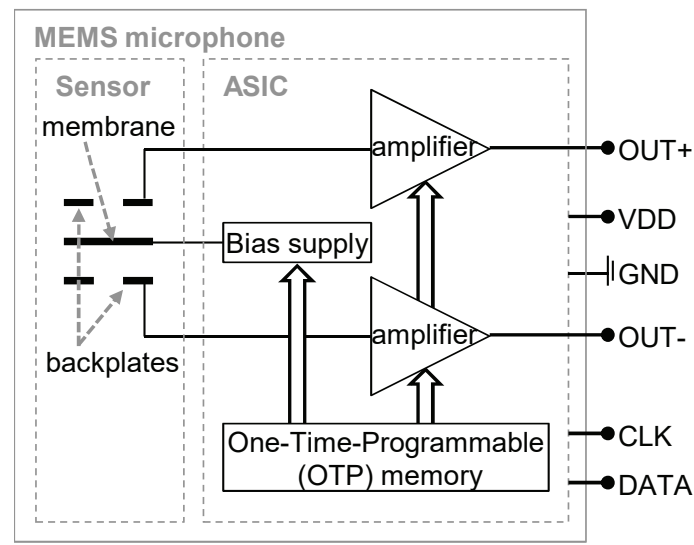

Fig. 2: Schematic of a programmable differential condenser MEMS microphone: differential sensor chip; programmable OTP module; adjustable bias supply module and amplifier stage.

The OTP memory allows adjusting the settings of the bias supply and the gain of the amplifier stages. Therefore, four bits of the memory cell are used to set the amplification and five bits of the memory cell are used to set the bias supply voltage. These two parameters directly influence the microphone output signal. The amplifier is part of the output stage of the ASIC and its programmable gain is designed within a range of $7.5 \mathrm{~dB}$ at a step size of $0.5 \mathrm{~dB}$. The microphone output signal and its harmonics are amplified linearly as long as the output stage does not contribute to nonlinearity. With this assumption, the microphone sensitivity can be shifted linearly by adjusting the gain factor with a slope of $1.0 \mathrm{dBV} / \mathrm{dB}$ as shown in Figure 3.

The variable bias voltage is designed within a range of $2.4 \mathrm{~V}$. For a precise trimming option a step size of $0.08 \mathrm{~V}$ was selected. Depending 
on the sensor and ASIC design, the operable bias range can be set from $9.8 \mathrm{~V}$ to $12.3 \mathrm{~V}$.

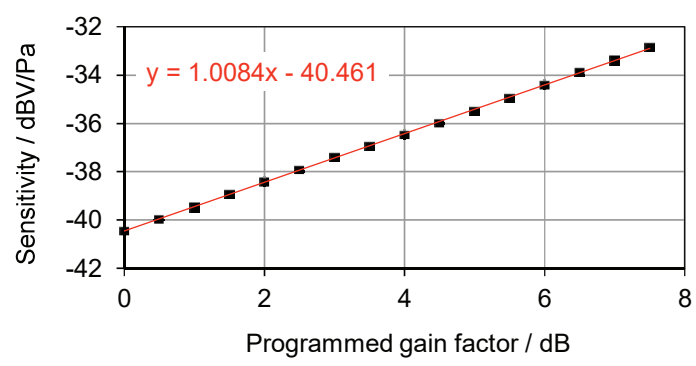

Fig. 3: $\quad$ Measured microphone sensitivity as a function of the programmed gain factors: linear sensitivity shift with a slope factor of $1.0 \mathrm{dBV} / \mathrm{dB}$.

Sound pressure deflects the membrane of a microphone. The forces which act on the membrane are first the mechanical force, which is opposed to the membrane deflection, and second the electrostatic forces. The electrostatic forces are caused by the field between the membrane and the two backplates. If the resulting electrostatic force is larger than the mechanical force, the membrane collapses onto one backplate. This is called pull-in. The nonlinear influence of the sensor system is small as long as the membrane deflection is far away from the pullin point. The pull-in can be reached by increasing the bias voltage as well as by increasing the sound pressure. Detailed investigations concerning differential MEMS microphones and their nonlinearity are shown in [11] and [13]. With the assumption of an operable bias range far away from the sensor pull-in point the microphone sensitivity can be shifted linearly by adjusting the bias voltage with a slope of $1.6 \mathrm{dBV} / \mathrm{V}$ as shown in Figure 4.

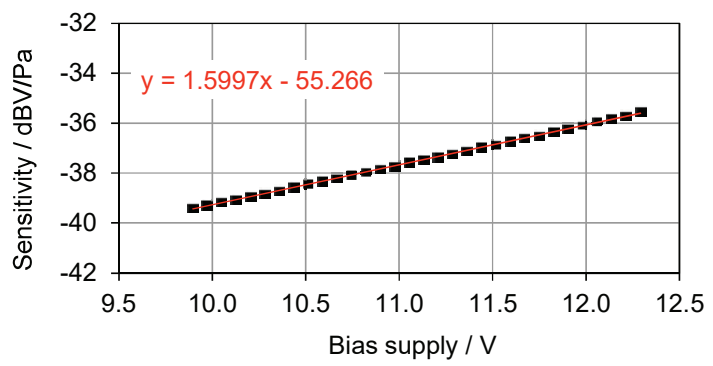

Fig. 4: $\quad$ Measured microphone sensitivity in relation to the bias voltages: linear sensitivity shift with a slope factor of $1.6 \mathrm{dBVN}$.
The influence of programming on the frequency response should be linear within the acoustical range up to $10 \mathrm{kHz}$. Figure 5 shows the difference of the sensitivities for maximum and minimum programmed gain and bias factors over the frequency. The sensitivity values were normalized to $0 \mathrm{dBV} @ 1 \mathrm{kHz}$. A detailed view of the frequency response of a flip-chip MEMS microphone is given in [9] and [14].

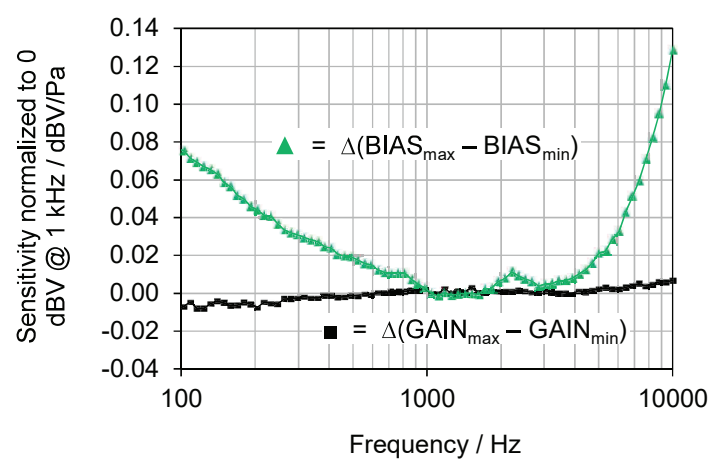

Fig. 5: Difference of the sensitivities for maximum and minimum programmed gain and bias factors over the frequency, normalized to $0 d B V @ 1 \mathrm{kHz}$.

In general, the frequency response depends on the MEMS microphone design, especially on the sensor chip and the package design. The frequency response is influenced by the microphone resonance at high frequencies and by the microphone cut-off behavior (high pass) for low frequencies. Trimming gain does not influence the frequency response. Trimming the bias voltage slightly influences the frequency response. However, the sensitivity variation over the frequency is small compared to the specified sensitivity tolerance of $\pm 1 \mathrm{~dB}$.

This allows compensating the process tolerances by trimming bias and gain after completing the microphone manufacturing process. An example of a programmed sensitivity distribution of a MEMS microphone production lot is given in [8]. There, a production lot with a measured sensitivity span of $4 \mathrm{~dB}$ was programmed to a narrow sensitivity span of $0.6 \mathrm{~dB}$. The programming was done in the production test.

Drawbacks of programming are the complexity and size of the ASIC chip as well as the complexity of the test equipment. For the programming additional space for the OTP module, for the digital interface, for the communication path and for the external clock is necessary. Another drawback is a longer test time. For a complete programming cycle in 
the production test $100 \mathrm{~ms}$ are needed. This time includes measuring the initial sensitivity, calculating the bias and gain values for the required sensitivity as well as programming the calculated parameters. Afterwards, read-out of the written memory is done and the electroacoustic performance is measured.

As shown before programming is a way to achieving a narrow sensitivity distribution. Another advantage of microphone programming is that other electroacoustic parameters can be optimized. Depending on the requirements of the customer, either a high SNR or a low THD at high SPL can be achieved after production.

\section{Programming high SNR}

In addition to a narrow sensitivity distribution of $\pm 1 \mathrm{~dB}$, the SNR of the microphones can be improved by using gain and bias programming. Figure 6 shows the measured SNR for all possible bias and gain settings as a function of the gain factor.

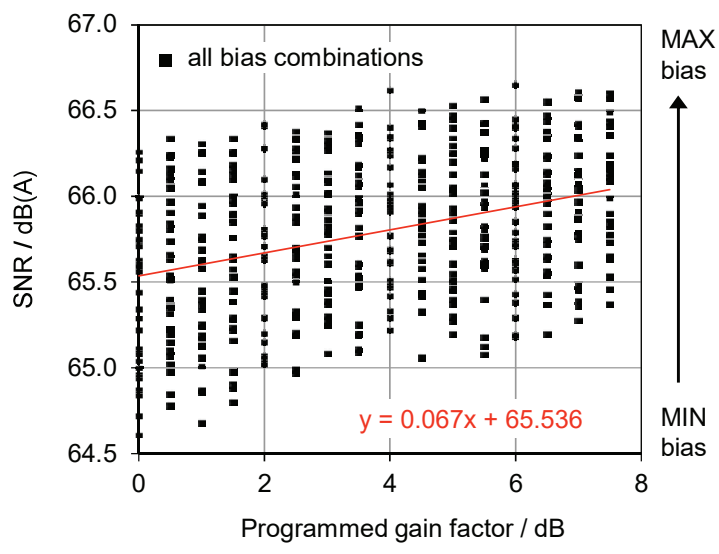

Fig. 6: $\quad$ Measured SNR for all possible bias and gain settings as a function of the gain factor.

As a result, the SNR is only slightly affected by programming gain, shown by a slope of 0.07 $\mathrm{dB}(\mathrm{A}) / \mathrm{dB}$. The small SNR influence is caused by the self-noise of the amplifier output stage as well as by the analog measurement input stage. The influence of the programmed bias voltages on the SNR is larger. An SNR range between $65.0 \mathrm{~dB}(\mathrm{~A})$ and $66.5 \mathrm{~dB}(\mathrm{~A})$ can be adjusted. Figure 7 shows the measured SNR for all possible bias and gain settings as a function of the programmed bias voltage. The SNR can be increased by programming bias voltages with a slope of around $0.5 \mathrm{~dB}(\mathrm{~A}) / \mathrm{V}$.

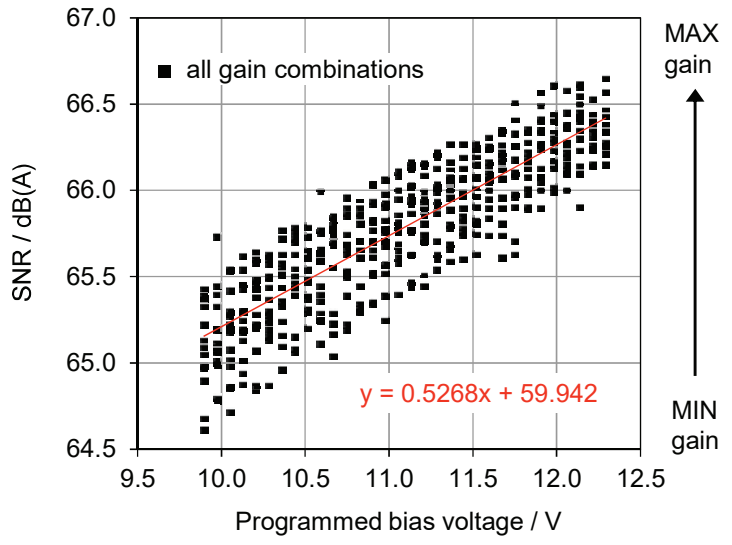

Fig. 7: $\quad$ Measured SNR for all possible bias and gain settings as a function of the programmed bias voltage.

In summary, the highest SNR can be reached by programming maximum bias voltages. A narrow sensitivity distribution could also be reached by programming the gain factor. A drawback of this is the rough gain trimming step size of $0.5 \mathrm{~dB}$. So, for a narrower sensitivity distribution, the rough gain calibration could be compensated by precise bias regulation. In general, higher bias voltages lead to higher SNR.

\section{Programming low THD at high SPL}

A drawback of programming high bias voltages are higher THDs. Increasing the bias voltage shifts the operating point of the MEMS sensor closer to the pull-in point, which increases the nonlinear behavior. This influences the overall microphone performance including THD.

The THD at high SPL can also be reduced by programming. Figure 8 shows microphone output signal and THD as functions of the SPL for two different bias voltages. The output signal $(\bullet, \square)$ and $\operatorname{THD}(\Delta, \diamond)$ increase with increasing SPL. The slightly nonlinear signal behavior results in a huge increase of the THD, shown in the last measurement points close to pull-in. A change of the bias voltage causes a shift of the signal and the THD curve parallel to the SPL axis. By increasing the bias voltages, the pull-in points are shifted to lower sound pressure levels.

For applications where high sound pressure recording is necessary, a lower bias voltage should be used. Therefore, higher sound pressures (here: $5 \mathrm{~dB}_{\mathrm{SPL}}$ ) without pull-in are possible for the same geometrical sensor design. 


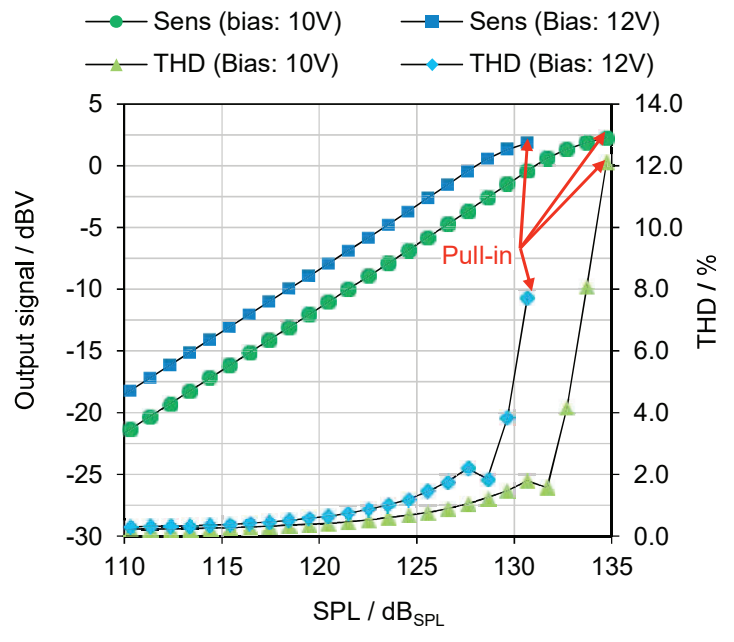

Fig. 8: Measured sensitivity and THD as a function of SPL for two different bias voltages.

The gain factor does not influence the THD as long as the signal is not influenced by the output stage full-scale range. Figure 9 shows the measured THD at $110 \mathrm{~dB}_{\mathrm{SPL}}$ for all possible gain and bias combinations as a function of the bias voltage.

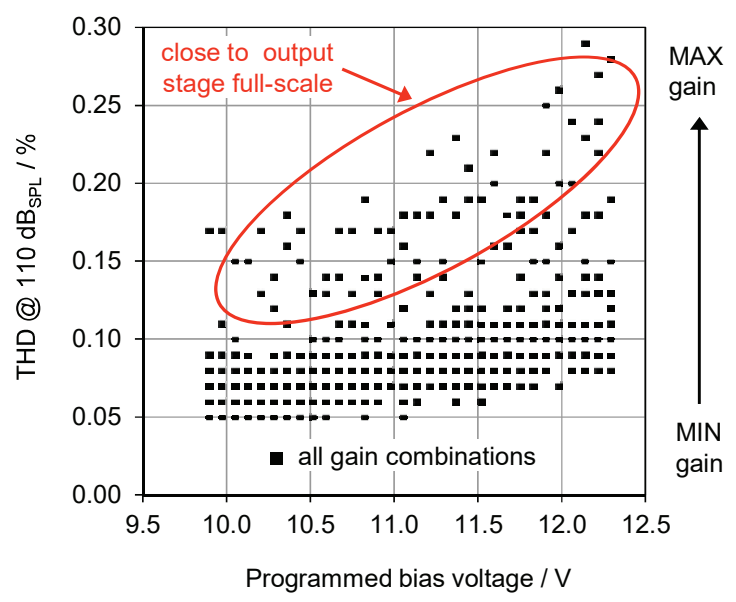

Fig. 9: Measured THD at $110 d B_{S P L}$ for all possible gain and bias combinations as a function of the bias voltage.

High gain factors increase the signal harmonics due to clipping. For lower sound pressure levels and lower bias voltages, the signal harmonics decrease. To extend the microphones dynamic range to higher sound pressures one possibility is to reduce the sensitivity, by programming low bias voltages. This allows shifting the pull-in point to higher sound pressure levels and reducing the THD. Tuning to a required sensitivity can be done by trimming the gain factor to a value below its full-scale.
For a high SPL setting bias voltages should be low and the gain factor small enough to avoid clipping of the signal. The drawback of decreasing the THD at high SPL is a reduction of SNR.

\section{Results: High SNR and high SPL setting}

With the knowledge of the influence of bias and gain on the acoustic performance it is possible to trim identical microphones towards two different specifications. Figure 10 shows all possible combinations of SNR and sensitivity, which can be realized by programming.

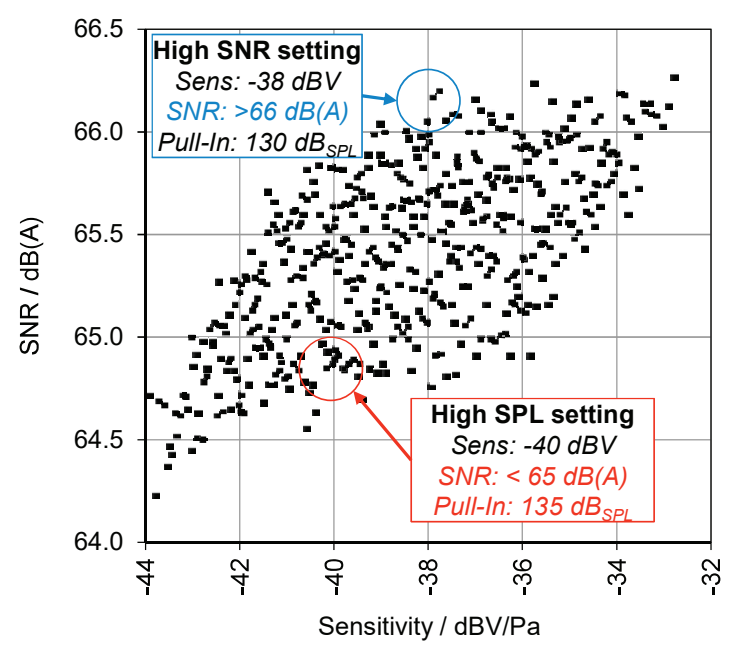

Fig. 10: SNR and Sensitivity for all possible programming combinations.

A high SNR (O) can be reached by programming a high bias voltage and shifting the sensitivity to a specified value by the gain setting. Here, an SNR of $>66 \mathrm{~dB}(\mathrm{~A})$ for a specified sensitivity of $-38 \mathrm{dBV}$ was reached. The pull-in point for this setting is around $130 \mathrm{~dB}_{\text {SPL. Low THD }}$ at high SPL (O) can be reached by programming a low bias voltage. An additional improvement is possible by reducing the sensitivity to a value of $-40 \mathrm{dBV}$. This results in an $\mathrm{SNR}<65 \mathrm{~dB}(\mathrm{~A})$ and a pull-in point at around $135 \mathrm{~dB}_{\mathrm{SPL}}$.

In the following, the benefits of the applicationspecific trimming are shown for whole production lots. Figure 11 shows a sensitivity distribution of a MEMS microphone production batch, measured in a production test. 


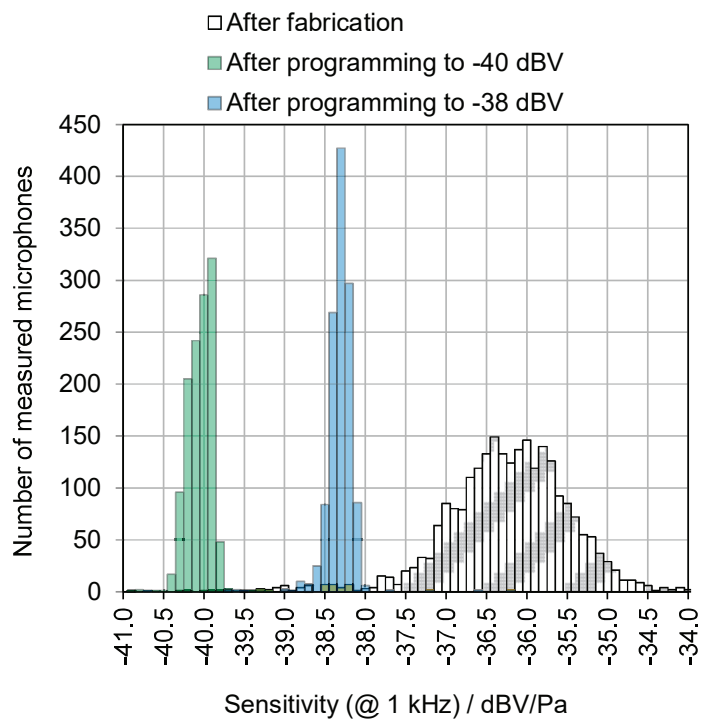

Fig. 11: Sensitivity distribution of a measured MEMS microphone production batch: after fabrication (口), after programming to $-40 \mathrm{dBV}$ "high SPL" ( $)$ and after programming to -38 dBV "high SNR" (ロ).

After the microphone fabrication, $(\square)$ the sensitivity distribution is measured with a mean sensitivity value of $-36.3 \mathrm{dBV} / \mathrm{Pa}$ and $\mathrm{a}$ standard deviation of $0.78 \mathrm{~dB}$. This standard deviation was reduced by programming. Therefore, a high SNR ( $\square$ ) and a high SPL ( $\square$ ) setting was used. The high SNR setting was programmed towards a mean sensitivity of $-38.4 \mathrm{dBV} / \mathrm{Pa}$. The standard deviation was reduced to a value of $0.18 \mathrm{~dB}$. The high SPL setting was programmed towards a mean sensitivity of $-40.1 \mathrm{dBV} / \mathrm{Pa}$. The standard deviation was reduced to a value of $0.15 \mathrm{~dB}$. Figure 12 shows the measured SNR distribution for the two different programmed settings, high SNR ( $\square$ ) and high SPL ( $\square$ ).

For the high SNR setting, the SNR distribution has a mean SNR value of $65.6 \mathrm{~dB}(\mathrm{~A})$ with a standard deviation of $0.72 \mathrm{~dB}$. The high SPL setting gives a distribution with a mean SNR of $65.0 \mathrm{~dB}(\mathrm{~A})$ and a standard deviation of $0.60 \mathrm{~dB}$. In summary, the SNR can be increased by around $1 \mathrm{~dB}$ for a MEMS microphone production batch by programming towards high SNR.

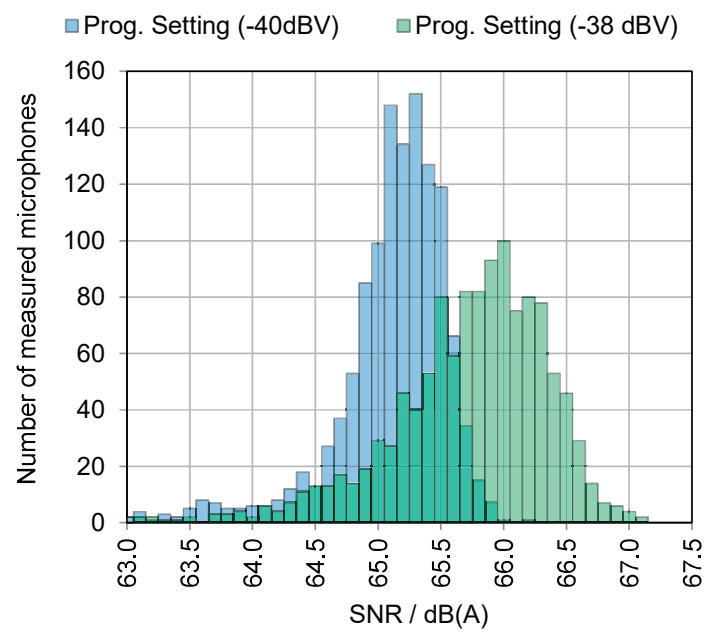

Fig. 12: Measured SNR distribution for the two different programmed settings, high SNR ( $\square$ ) and high SPL ( $\square$ ).

In contrast to the high SNR setting, the high SPL setting leads to smaller THD at high SPL. For SPL of $127 \mathrm{~dB}_{\mathrm{SPL}}$ the measured THD value was reduced from $2.2 \%$ (high bias voltages) to $1.0 \%$ (low bias voltages), as shown before in Figure 8.

\section{Conclusions}

Programmable MEMS microphones allow the trimming of the acoustic parameters after the fabrication process. The microphones can be tuned towards the specific requirements of the application. In addition to a narrow sensitivity distribution, the SNR and THD performance can be improved by tuning the bias voltages and the gain factors.

A high SNR, which is necessary for high-end recording of speech and music, can be reached by increasing the bias voltage. An SNR of up to $66.5 \mathrm{~dB}(\mathrm{~A})$ was reached in an experiment with one production lot. The gain factor does not influence the SNR significantly. It is used to trim the microphones sensitivity to a specified value. In mass production the SNR can be increased by $1 \mathrm{~dB}(\mathrm{~A})$ with a narrow sensitivity distribution of $\pm 0.5 \mathrm{dBV}$ by programming towards a high SNR.

A low THD at high SPL is necessary for recording in high SPL environments like rock concerts. Low THD at high SPL can be reached by low bias voltages. With such a high SPL setting the measured THD @ $127 \mathrm{~dB}_{\mathrm{SPL}}$ has the value of $1.0 \%$ in comparison to $2.2 \%$ for the high SNR setting.

In summary, programmable MEMS microphones from the same fabrication lot can be trimmed to different specifications. Without 
changing the geometries of the sensor chip, the microphone can be tuned to a high SNR or a low THD application.

\section{Literature}

[1] J.W. Weigold, T.J. Brosnihan, J. Bergeron, X. Zhang, A MEMS Condenser Microphone For Consumer Applications, Proceedings of Micro Electro Mechnical Systems, 2006, pp. 86-89; doi: 10.1109/MEMSYS.2006.1627742.

[2] G. Feiertag, M. Winter, A. Leidl, Flip chip packaging for MEMS microphones, Journal of Microsystem Technologies, vol 16, 2010, pp. 817-823, doi: 10.1007/s00542-010-1039-3.

[3] M. Fueldner, A. Dehe, R. Lerch, Analytical Analysis and Finite Element Simulation of Advanced Membranes for Silicon Microphones, IEEE Sensor Journal, vol. 5, no. 5, 2005, pp. 857-863, doi: 10.1109/JSEN.2004.841449.

[4] D.T. Martin, J. Liu, K. Kadirvel, R.M. Fox, M. Sheplak, T. Nishida, A Micromachined DualBackplate Capacitive Microphone for Aeroacoustic Measurements, Journal of Microelectromechanical Systems, vol. 16, no. 6, 2007, pp. 1289-1302, doi: 10.1109/JMEMS.2007.909234.

[5] W. Conklin, Leveraging microelectromechanical microphones inherent matching to reduce noise using multiple microphone elements, Proceedings of Meetings on Acoustics, vol. 19, 2013, doi: 10.1121/1.4799235.

[6] P.R. Scheeper, A.G.H. Donk, W. Olthuis, P. Bergveld, A review of silicon microphones, Journal of Sensors and Actuators A 44, 1994, pp. 1-11, doi: 10.1016/0924-4247(94)00790-X.

[7] Z.-H. Fu, F. Fan, J.-D. Huang, Dual-microphone noise reduction for mobile phone application, Proceedings of International Acoustic, Speech and Signal Processing, 2013, pp. 7239-7243, doi: 10.1109/ICASSP.2013.6639068.

[8] S. Walser, C. Siegel, M. Winter, A. Leidl, G. Feiertag, A Novel Method for Reducing the Sensitivity Deviation of MEMS Microphones by Programming, Procedia Engineering 120, 2015, pp.206-209, doi: 10.1016/j.proeng.2015.08.611.

[9] S. Walser, C. Siegel, M. Winter, M. Loibl, W. Pahl, A. Leidl, G. Feiertag, Flip-Chip MEMS Microphone Package With Small Front-Volume and Large Back-Volume, Proceedings of European Microelectronics and Packaging Conference, Friedrichshafen, Germany, Sept. 14-16, 2015

[10] B.-H. Kim, H.-S. Lee, Acoustical-Thermal Noise in a Capacitive MEMS Microphone, IEEE Sensors Journal, vol. 15, no. 12, 2015, pp. 6853-6860, doi: 10.1109/JSEN.2015.2464372.

[11]D.T. Martin, Design, fabrication and characterization of a MEMS dual-backplate capacitive microphone, Dissertation, 2007, University of Florida, USA.

[12] R.S.C. Wang, R.S.J. Shen, C.C.H. Hsu, Neobithigh reliable logic non-volatile memory (NVM), Proceedings $11^{\text {th }}$ International Symposium on the Physical and Failure Analysis of Integrated
Circuits, 2004, pp. 11-114, doi: 10.1109/IPFA.2004.1345560.

[13] J. Liu, Nonlinear Dynamics of a Dual-Backplate Capacitive MEMS Microphone, Dissertation, 2007, University of Florida, USA.

[14] M. Winter, G. Feiertag, A. Leidl, H. Seidl, Influence of a chip scale package on the frequency response of a MEMS microphone, Microsystem Technologies, vol. 16, 2010, pp. 809-815, doi: 10.1007/s00542-009-0994-z. 